\title{
Single-mode laser action in quantum cascade lasers with spiral-shaped chaotic resonators
}

\section{Citation}

Audet, Ross, Mikhail A. Belkin, Jonathan A. Fan, Benjamin G. Lee, Kai Lin, Federico Capasso, Evgenii E. Narimanov, et al. 2007. "Single-Mode Laser Action in Quantum Cascade Lasers with Spiral-Shaped Chaotic Resonators." Applied Physics Letters 91 (13): 131106. https:// doi.org/10.1063/1.2784290.

\section{Permanent link}

http://nrs.harvard.edu/urn-3:HUL.InstRepos:41371595

\section{Terms of Use}

This article was downloaded from Harvard University's DASH repository, and is made available under the terms and conditions applicable to Other Posted Material, as set forth at http:// nrs.harvard.edu/urn-3:HUL.InstRepos:dash.current.terms-of-use\#LAA

\section{Share Your Story}

The Harvard community has made this article openly available. Please share how this access benefits you. Submit a story.

Accessibility 


\title{
Single-mode laser action in quantum cascade lasers with spiral-shaped chaotic resonators
}

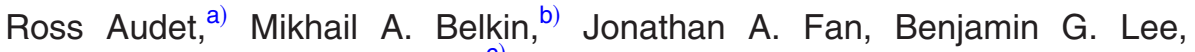 \\ Kai Lin, and Federico Capasso ${ }^{\mathrm{C})}$ \\ Harvard School of Engineering and Applied Sciences, Harvard University, Cambridge, \\ Massachusetts 02138, USA \\ Evgenii E. Narimanov \\ School of Electrical and Computer Engineering, Purdue University, West Lafayette, Indiana 47907, USA \\ David Bour, ${ }^{\text {d) }}$ Scott Corzine, ${ }^{\text {e) }}$ Jintian Zhu, and Gloria Höfler ${ }^{\text {f) }}$ \\ Agilent Laboratories, 3500 Deer Creek Road, Palo Alto, California 94304, USA
}

(Received 18 June 2007; accepted 23 August 2007; published online 25 September 2007)

\begin{abstract}
The authors have fabricated and characterized quantum cascade lasers with spiral-shaped microresonators. The lasers operate in pulsed mode at room temperature with peak optical power greater than $20 \mathrm{~mW}$ and in continuous wave at temperatures up to $125 \mathrm{~K}$. They exhibit single-mode emission in both pulsed mode and continuous wave operation, with a $30 \mathrm{~dB}$ side-mode suppression ratio at injection currents well above threshold. Subthreshold spectral measurements indicate that the spiral cavities support whispering-gallery-like modes. Single-mode lasing occurs on one of these modes. Far-field profiles reveal enhanced directionality compared to microdisk lasers. (c) 2007 American Institute of Physics. [DOI: 10.1063/1.2784290]
\end{abstract}

Microcavity lasers have many potential applications because of their low threshold currents and suitability for highdensity integration. Certain microcavities, such as microcylinders, support high- $Q$ whispering gallery (WG) modes, but the output power of lasers based on such geometries is negligible. The power and directionality of microcylinder quantum cascade lasers can be improved by orders of magnitude by deforming the cavities to support "bow-tie modes." Spiral-shaped cavities with "notch" discontinuities [see Fig. 1] have also been examined as a means of achieving high-power directional emission from optically pumped polymer lasers $^{2}$ and electrically pumped InGaN multiple-quantum-well ${ }^{3}$ lasers. Light in WG-like modes is outcoupled in these structures by diffraction at the spiral notch. $^{4}$

In this work, we have processed and characterized midinfrared quantum cascade lasers (QCLs) with spiral microcavities. These devices exhibit high-power emission and a strong tendency toward single-mode operation over a wide range of injection currents.

The active region of our QCLs consists of 30 stages of a "three-quantum-well" active region design ${ }^{5}$ with target wavelength of $8 \mu \mathrm{m}$, grown by metal organic vapor phase epitaxy. ${ }^{6}$ The waveguide layers are similar to those in Ref. 6. Emission in the midinfrared permits a smaller ratio of cavity size to mode wavelength and a concomitant reduction of scattering due to surface roughness compared to previously reported spiral-shaped lasers emitting at visible wavelengths.

\footnotetext{
${ }^{a}$ Present address: Department of Electrical Engineering, Stanford Univer sity, Stanford, CA 94350. Electronic mail: ross.audet@post.harvard.edu

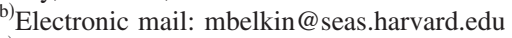

${ }^{c)}$ Electronic mail: capasso@seas.harvard.edu

d) Present address: Photodigm, Richardson, Texas 75081.

e) Present address: Infinera, Sunnyvale, CA 94089.

${ }^{\mathrm{f})}$ Present address: Argos Tech, Santa Clara, CA 95051.
}

The top profile of our spiral microlasers was defined by the expression $r(\phi)=r_{0}+\Delta r \phi / 2 \pi$ [see Fig. 1]. Lasers were fabricated with $r_{0}=50,80$, and $110 \mu \mathrm{m}$. For each radius, two deformations were tested, $\Delta r=7 \mu \mathrm{m}$ and $\Delta r=10 \mu \mathrm{m}$. The $15 \mu \mathrm{m}$ high spiral pillars with vertical walls were formed in QCL material by inductively coupled plasma reactive ion etching $\left(\mathrm{HBr}: \mathrm{BCl}_{3}: \mathrm{Cl}_{2}, 10: 5: 2\right)$ using a Microposit SU-8 2005 photoresist mask. Top metal contacts (Ti/Au, $10 \mathrm{~nm} / 150 \mathrm{~nm}$ ) were positioned on top of the pillars. The substrate was then thinned to $200 \mu \mathrm{m}$, and a Ge/Au $(20 \mathrm{~nm} / 200 \mathrm{~nm})$ back contact was deposited. Cleaved devices were soldered with indium to a copper block and electrically connected via wire bonds at the top of the spiral micropillars.

Room temperature (RT) lasing in pulsed mode was achieved for all spiral sizes and deformations. The voltage

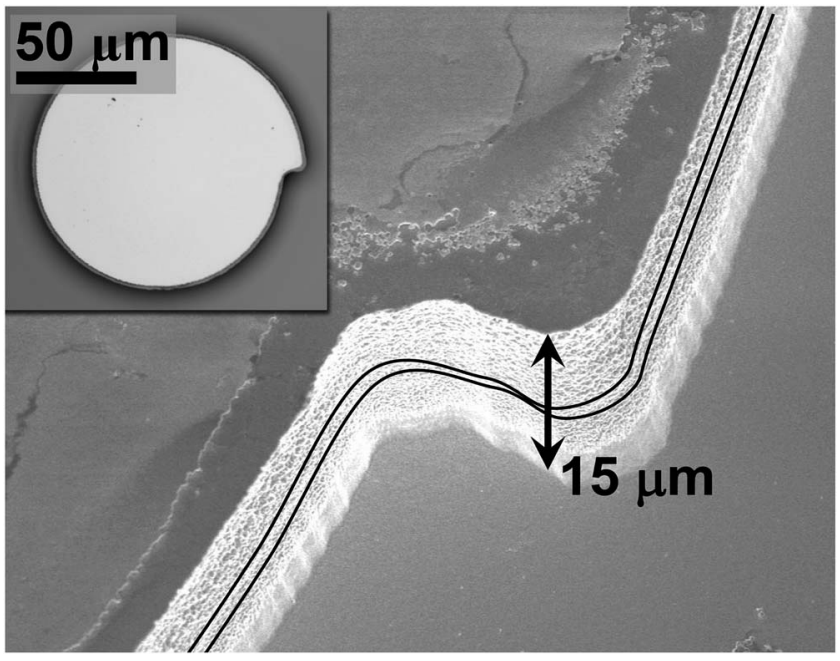

FIG. 1. Scanning electron microscope image of the spiral notch in a fabricated device; the position of the active region is indicated by two black lines. Inset: optical microscope image of the device. 

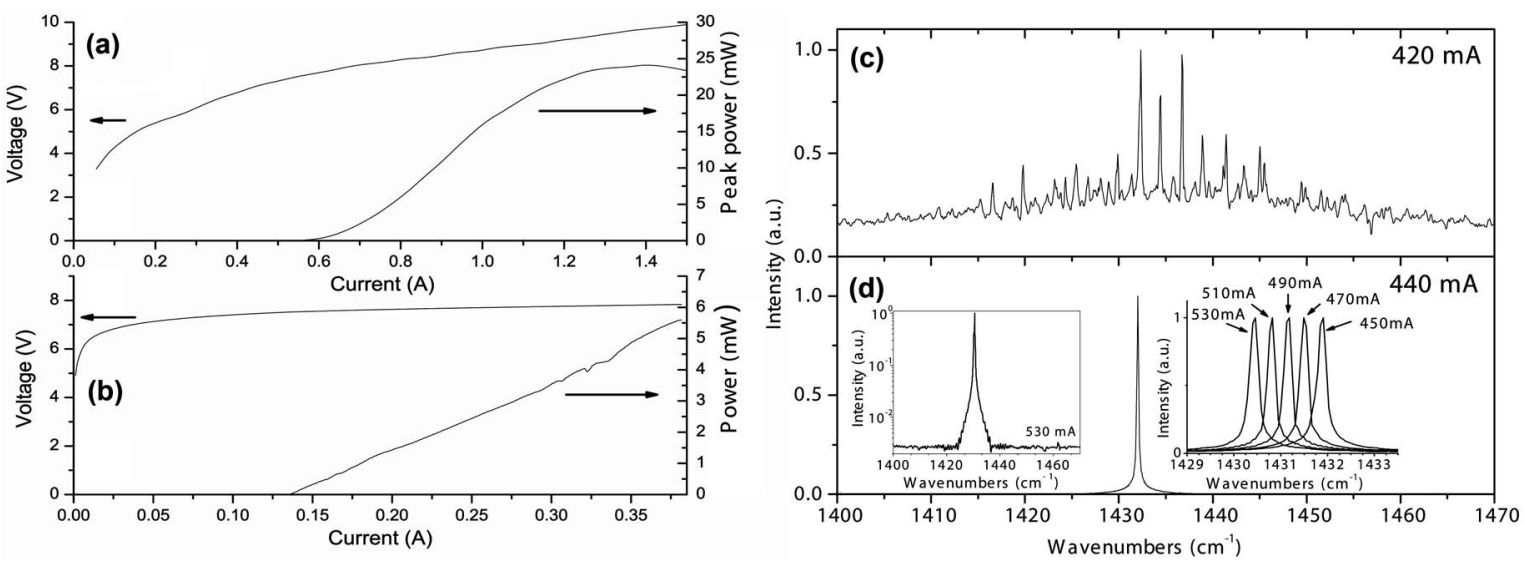

FIG. 2. (a) Voltage and peak optical power as a function of peak injection current for a device with $r_{0}=80 \mu m$ and $\Delta r=10 \mu m$, operated in pulsed mode ( 80 $\mathrm{kHz} 0.5 \%$ duty cycle) at room temperature. (b) Voltage and optical power as a function of injection current for a device with $r_{0}=80 \mu m$ and $\Delta r=7 \mu m$ operated in continuous wave at $77 \mathrm{~K}$. Optical power data in (a) and (b) are not corrected for the collection efficiency. (c) and (d) Continuous wave emission spectra obtained at $77 \mathrm{~K}$ for a representative device with $r_{0}=110 \mu \mathrm{m}$ and $\Delta r=10 \mu \mathrm{m}$. The threshold current is $430 \mathrm{~mA}$. (c) Subthreshold amplified spontaneous emission spectrum at $420 \mathrm{~mA}$ current. We have identified two interleaved sets of peaks obscured by water absorption lines: one set with peak positions at approximately $1429.9,1434.4,1438.9,1443.3 \mathrm{~cm}^{-1}$ and another one with peak positions at approximately $1432.3,1436.8,1441.3,1445.3 \mathrm{~cm}^{-1}$. (d) Singlemode laser emission at $440 \mathrm{~mA}$ current. Left inset: logarithmic scale plot showing single-mode laser emission at $530 \mathrm{~mA}$ current. Right inset: shift in single-mode emission wavelength as a function of pump current.

and optical power versus current for a representative device with $r_{0}=80 \mu \mathrm{m}$ and $\Delta r=10 \mu \mathrm{m}$ are shown in Fig. 2(a). Power measurements were performed using a polished metal tube to channel the radiation emitted by the device onto a calibrated thermopile detector. From far-field measurements (discussed below), we estimate the optical power collection efficiency of this method to be $\sim 50 \%$. The slope efficiency of the device in Fig. 2(a) was approximately $40 \mathrm{~mW} / \mathrm{A}$, not corrected for collection efficiency. This is three to four times smaller than the 120-180 mW/A per facet reported in Refs. 5 and 6 for ridge waveguide lasers processed from a similar material. The spiral device of Fig. 2(a) exhibited a threshold current density of $3 \mathrm{kA} / \mathrm{cm}^{2}$. For a device of the same radius with a smaller notch of $\Delta r=7 \mu \mathrm{m}$, a RT threshold current density of $2.25 \mathrm{kA} / \mathrm{cm}^{2}$ was observed. This is lower than the value of $2.75 \mathrm{kA} / \mathrm{cm}^{2}$ reported in Ref. 6 for ridge waveguide lasers processed from a similar material.

Continuous wave (cw) operation was demonstrated at temperatures up to $125 \mathrm{~K}$. At this temperature, the bond wires were destroyed by Ohmic heating, indicating that higher temperature operation may be possible with thicker wires. At $77 \mathrm{~K}$, devices with $r_{0}=80 \mu \mathrm{m}$ and $\Delta r=10 \mu \mathrm{m}$ had a threshold current density of approximately $850 \mathrm{~A} / \mathrm{cm}^{2}$. A device with the same radius and a smaller notch $(\Delta r$ $=7 \mu \mathrm{m})$ exhibited a lower threshold of $650 \mathrm{~A} / \mathrm{cm}^{2}$. The voltage and optical power versus current for this device operated cw at $77 \mathrm{~K}$ are shown in Fig. 2(b). Optical power was collected using two ZnSe lenses: one to collect light from the device and another to refocus it onto the detector. We estimate a collection efficiency of approximately $7 \%$ for this method.

In both pulsed operation at RT and cw operation at $77 \mathrm{~K}$, the threshold current density for devices with $r_{0}=50 \mu \mathrm{m}$ and $\Delta r=10 \mu \mathrm{m}$ was approximately a factor of 2 larger than that for devices with $r_{0}=80 \mu \mathrm{m}$ and $\Delta r=10 \mu \mathrm{m}$. For devices with $r_{0}=110 \mu \mathrm{m}$ and $\Delta r=10 \mu \mathrm{m}$, it was measured to be approximately $40 \%$ smaller than that for devices with $r_{0}$ $=80 \mu \mathrm{m}$ and $\Delta r=10 \mu \mathrm{m}$. In general, devices with smaller deformation ratio $\Delta r / r_{0}$ had lower threshold current densities. densities.
Downloaded 26 Sep 2007 to 128.103 .60 .225 . Redistribution subject to AlP license or copyright,
Emission spectra for the various devices were collected with a Fourier transform infrared spectrometer equipped with a liquid nitrogen-cooled mercury cadmium telluride (MCT) detector. To gain insight into the resonances supported by the spiral-shaped cavities, the lasers were driven slightly below threshold in $\mathrm{cw}$ at $77 \mathrm{~K}$, and amplified spontaneous emission (ASE) spectra were measured [see Fig. 2(c)]. The ASE spectrum in Fig. 2(c) for a device with $r_{0}=110 \mu \mathrm{m}$ and $\Delta r$ $=10 \mu \mathrm{m}$ reveals two interspersed sets of peaks, with each set exhibiting mode spacing of approximately $4.5 \mathrm{~cm}^{-1}$, the expected spacing between WG modes in a circular device of the same radius. The two sets of peaks, distinguished by their relative magnitudes, likely result from the degeneracy between clockwise- and counterclockwise-propagating WGlike modes being removed by the spiral notch. ASE spectra with mode spacing strongly indicative of WG-like modes were also obtained for devices with $r_{0}=80 \mu \mathrm{m}$ and $50 \mu \mathrm{m}$.

Despite the close spacing of the cavity modes relative to the gain bandwidth (full width at half maximum

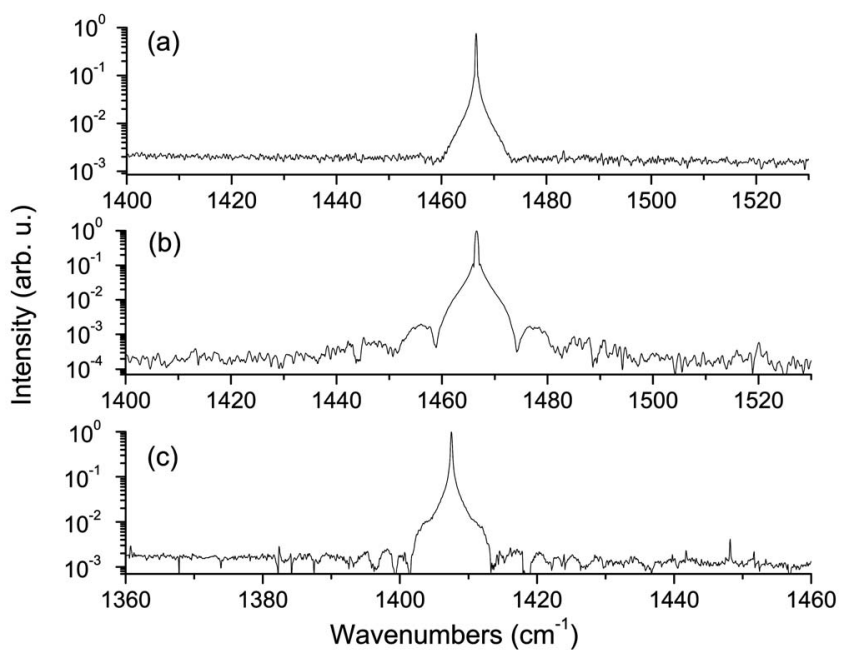

FIG. 3. Emission spectra in pulsed mode showing single-mode operation. (a,b) Representative device with $r_{0}=80 \mu \mathrm{m}$ and $\Delta r=7 \mu \mathrm{m}$ at $77 \mathrm{~K}$, peak currents of (a) $165 \mathrm{~mA}$, (b) $350 \mathrm{~mA}$. (c) Data for the device of Fig. 2(a) at 


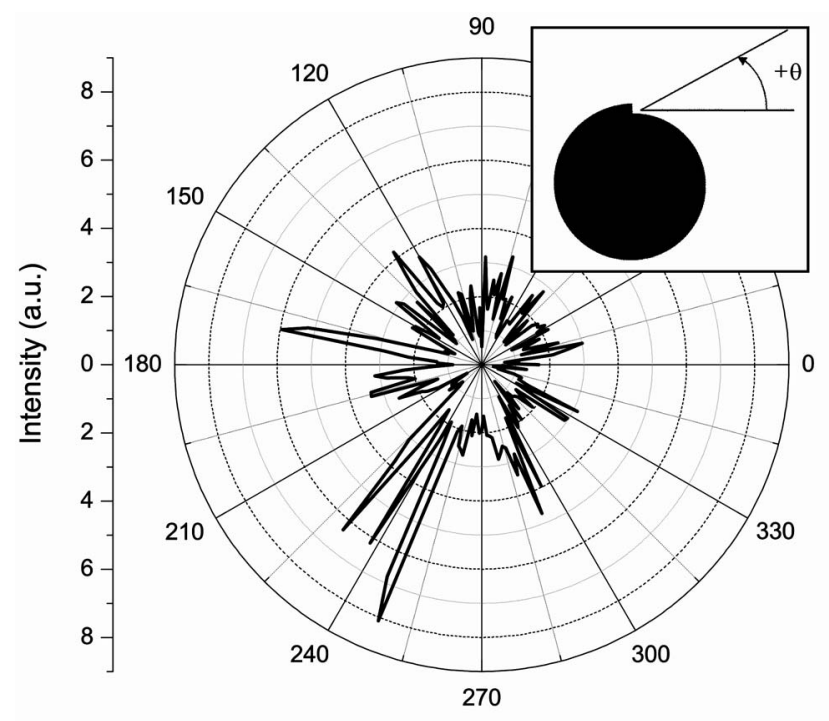

FIG. 4. Far-field profile of representative device with $r_{0}=80 \mu \mathrm{m}$ and $\Delta r$ $=10 \mu \mathrm{m}$, operated in pulsed mode at room temperature at peak current of $1500 \mathrm{~mA}$ (threshold current is $800 \mathrm{~mA}$ ). Zero angle is defined as perpendicular to the spiral notch.

$>100 \mathrm{~cm}^{-1}$ ), laser emission in both $\mathrm{cw}$ and pulsed operation is typically strongly single mode, with a side-mode suppression ratio (SMSR) near $30 \mathrm{~dB}$, even at currents significantly above threshold [see Fig. 2(d)]. In single-mode operation, lasing occurs on one of the cavity modes found in the ASE spectrum [see Figs. 2(c) and 2(d)]. For the device shown in Fig. 2(d), cw emission at $77 \mathrm{~K}$ is single mode from the threshold current of 430 up to $530 \mathrm{~mA}$. The laser operated without mode hopping across this range of injection currents, with a wavelength tuning coefficient of $-1.75 \mathrm{~cm}^{-1} / \mathrm{A}$ [see the inset to Fig. 2(d)]. Similar behavior is observed in pulsed operation; spectra from a representative device at $77 \mathrm{~K}$ reveal single-mode emission with nearly $30 \mathrm{~dB}$ SMSR over a range of injection currents from $145 \mathrm{~mA}$ (threshold) to more than $350 \mathrm{~mA}$ [see Fig. 3(a) and 3(b)] At RT, single-mode operation was demonstrated at currents up to $40 \%$ above threshold [see Fig.3(c)]. To compare this behavior with conventional ridge waveguide lasers with similar cavity mode spacing, we collected emission spectra from 370- $\mu$ m-long ridge lasers processed from the same QCL material. In both pulsed mode and $\mathrm{cw}$ operation, the ridge lasers were multimode even slightly $(<10 \%)$ above threshold, exhibiting Fabry-Perot mode spacing of $4.2 \mathrm{~cm}^{-1}$ that corresponded to their cavity lengths.

We attribute the tendency to single-mode lasing of our spiral lasers to a reduction in spatial hole burning (SHB). In ridge waveguide QCLs, the standing Fabry-Perot waves result in SHB which causes multimode lasing. ${ }^{7}$ The standing wave, formed by a cavity mode, imprints a grating in the gain medium through gain saturation; as a result, other modes become more favorable for lasing, and multimode operation is triggered. This process is favored in QCLs by the short gain recovery time compared to the gain grating diffusion time.

In devices with WG resonances, however, SHB is suppressed because the laser modes are traveling waves. We note that single-mode emission has previously been reported for a number of WG microdisk QCLs, ${ }^{8,9}$ supporting this explanation. The spiral resonator appears to preserve WG-like modes while increasing output coupling.

The chaotic dynamics of the spiral resonator may further contribute to suppressing SHB as spiral devices with the largest deformation parameter $\Delta r / r_{0}$ tend to show singlemode performance over the widest range of injection currents. Other chaotic effects, such as dynamical localization ${ }^{10}$ and quasi scarred resonances, ${ }^{11}$ could also play an important role in determining mode structure and mode selection.

The radial far-field profiles of several devices were also examined [see Fig. 4]. A single device was cleaved and soldered to a copper pedestal atop a rotating stage, providing unobstructed optical access. A MCT detector with a sensor size of 250 by $250 \mu \mathrm{m}$ was placed $2 \mathrm{~cm}$ from the device in the plane of the spiral, and the signal was recorded as a function of $2^{\circ}$ increments of rotation. The profile in Fig. 4 reveals several sharply defined lobes. The locations of peaks were generally independent of pump current above threshold.

The far-field profiles of our spiral QCLs are markedly different than those of Ref. 3 for InGaN lasers, where directional emission into an approximately $60^{\circ}$ angle was observed. We believe the dissimilarity results partly from the larger ratio of emission wavelength to spiral radius in our devices. Additionally, the higher refractive index of our material system creates differing conditions for refraction and diffractive escape.

Devices were processed in the Center for Nanoscale Systems (CNS) at Harvard University. Harvard-CNS is a member of the National Science Foundation, National Nanotechnology Infrastructure Network.

${ }^{1}$ C. Gmachl, F. Capasso, E. E. Narimanov, J. U. Nöckel, A. D. Stone, J. Faist, D. L. Sivco, and A. Y. Cho, Science 280, 1556 (1998).

${ }^{2}$ T. Ben-Messaoud and J. Zyss, Appl. Phys. Lett. 86, 241110 (2005).

${ }^{3}$ M. Kneissl, M. Teepe, N. Miyashita, N. M. Johnson, G. D. Chern, and R. K. Chang, Appl. Phys. Lett. 84, 2485 (2004).

${ }^{4}$ F. Courvoisier, V. Boutou, J. P. Wolf, R. K. Chang, and J. Zyss, Opt. Lett. 30, 738 (2005).

${ }^{5}$ C. Gmachl, A. Tredicucci, F. Capasso, A. L. Hutchinson, D. L. Sivco, J. N. Baillargeon, and A. Y. Cho, Appl. Phys. Lett. 72, 3130 (1998).

${ }^{6}$ M. Troccoli, D. Bour, S. Corzine, G. Höfler, A. Tandon, D. Mars, D. J. Smith, L. Diehl, and F. Capasso, Appl. Phys. Lett. 85, 5842 (2004).

${ }^{7}$ C. Y. Wang, L. Diehl, A. Gordon, C. Jirauschek, F. X. Kärtner, A. Belyanin, D. Bour, S. Corzine, G. Höfler, M. Troccoli, J. Faist, and F. Capasso, Phys. Rev. A 75, 031802 (2007).

${ }^{8}$ J. Faist, C. Gmachl, M. Striccoli, C. Sirtori, F. Capasso, D. L. Sivco, and A. Y. Cho, Appl. Phys. Lett. 69, 2456 (1996).

${ }^{9}$ C. Gmachl, J. Faist, F. Capasso, C. Sirtori, D. Sivco, and A. Y. Cho, IEEE J. Quantum Electron. 33, 1567 (1997).

${ }^{10}$ W. Fang, H. Cao, V. A. Podolskiy, and E. E. Narimanov, Opt. Express 13, 5641 (2005).

${ }^{11}$ S. Y. Lee, S. Rim, J. W. Ryu, T. Y. Kwon, M. Choi, and C. M. Kim, Phys. Rev. Lett. 93, 164102 (2004). 